\title{
Effect of inulin in the treatment of irritable bowel syndrome with constipation (Review)
}

\author{
OANA-BOGDANA BĂRBOI ${ }^{1,2}$, IRINA CIORTESCU ${ }^{1,2}$, IOAN CHIRILA $^{3}$, \\ CARMEN ANTON ${ }^{1,2}$ and VASILE DRUG ${ }^{1,2}$
}

\author{
${ }^{1}$ Department of Gastroenterology, 'Grigore T. Popa' University of Medicine and Pharmacy, \\ 700115 Iasi; ' ${ }^{2}$ nstitute of Gastroenterology and Hepatology, 'Saint Spiridon’ Hospital, 700111 Iasi; \\ ${ }^{3}$ Health Related to Environment Department, National Institute of Public Health, 700506 Iasi, Romania
}

Received June 15, 2020; Accepted July 15, 2020

DOI: $10.3892 / \mathrm{etm} .2020 .9315$

\begin{abstract}
At present, irritable bowel syndrome (IBS) is a common medical problem all over the world that implies considerable social burden and high costs. Considering the different pathophysiological pathways, unitary management for IBS is not possible. Of the therapeutic approaches that have been proposed so far, only a few have been demonstrated to have beneficial effects in IBS patients. The implication of gut microbiota in IBS is obvious, similarly to the therapeutic effect of pro-/prebiotics, which is reflected by the latest publications. The intake of inulin seems to regulate the bowel peristalsis and colonic transit, the consistency and frequency of the stools, as it changes the composition of gut microbiota. The beneficial effect of inulin in patients with IBS-constipation form (IBS-C) is obvious, but still, more randomized controlled clinical trials involving large samples of patients are needed in order to provide more evidence.
\end{abstract}

\section{Contents}

1. Introduction

2. Inulin and stool frequency

3. Inulin and stool consistency

4. Inulin and transit time

5. Inulin and patients satisfaction

6. Inulin and gut microbiota

7. Conclusion

Correspondence to: Dr Carmen Anton, Department of Gastroenterology, 'Grigore T. Popa' University of Medicine and Pharmacy, 16 Universitatii Street, 700115 Iasi, Romania

E-mail: carmen_ro2008@yahoo.com

Key words: inulin, irritable bowel syndrome, constipation, gut microbiota, prebiotic, probiotic

\section{Introduction}

The consumption of dietary fiber in the general population is still low despite the publicity, all the guidelines and recommendations $(1,2)$. Dietary fibers are defined by the Institute of Medicine Food Nutrition Board as 'non-digestible carbohydrates and lignin that are intrinsic and intact in plants' (1). Fibers are not digested until they reach the colon and they are fermented by friendly microbiota, being a reliable source of energy. These type of substances are considered as prebiotics, since they selectively stimulate beneficial bacteria and provide health benefits to the human body. Higher intakes of dietary fibers contribute to reduce the risk of developing several chronic diseases, including chronic constipation through modification of laxation and fermentation, and its effects on gut microbiota (1). The composition of microbiota adapts promptly as a result of dietary changes whereas we refer to dietary fiber and macronutrients intake or consumption of prebiotic fibers (3). Prebiotics such as inulin or fructo-oligosaccharides are characterized as 'functional fibers' (4).

Inulin is a nondigestible oligosaccharide that serves in the diet as a soluble fiber that is naturally found in more than 36,000 of species of plants, including vegetables such as wheat, garlic, onion, chicory, artichoke and asparagus. Due to its chemical configuration, inulin is stable to hydrolysis by digestive enzymes, so it reaches the colon undigested and is further selectively fermented by colonic microflora (5). It is commonly extracted from chicory roots for commercial use. Due to its physicochemical and nutritional properties inulin is exploited as a compound in various foods and drinks. In the United States in 2018, the Food and Drug Administration approved inulin as a dietary fiber ingredient used to improve the nutritional value of manufactured food products.

The intake of inulin has been linked to the regulation of bowel peristalsis and transit, stool consistency and frequency, as it produces changes from the composition and activity of the gut microbiota, to the modulation of the immune response, to the mineral absorption and also to the satiety and bone weight (6). Thus, inulin has beneficial effects on healthy individuals, as demonstrated in a trial published in 2007 (7). According to the study, the consumption of bakery products 
enriched with inulin stimulates the growth of bifidobacteria, which contributes to dissolution of pathogenic bacteria. Another study on healthy volunteers confirmed the prebiotic effect of fruits and vegetables containing inulin derived from Jerusalem artichoke (8).

Irritable bowel syndrome (IBS) is a common medical problem all over the world that implies substantial social burden and high costs. Its pathophysiology is still not completely understood, but it is considered as a disorder due to an alteration in the brain-gut axis. This alteration is responsible for abnormalities of autonomic, enteric or central nervous systems. As a consequence, altered gastrointestinal motility and permeability, disturbed immune response and disrupted intestinal microbiota occur and represent a trigger for IBS symptoms (9). Considering the different pathophysiological pathways, an unitary management for IBS is not possible. From all the therapeutic approaches that have been proposed so far, only a few have been demonstrated to have beneficial effects in IBS patients.

The implication of gut microbiota in IBS is obvious, as is the therapeutic effect of pro-/prebiotics, which is reflected by recent publications. The beneficial effect of inulin in patients with IBS-constipation form (IBS-C) is obvious, but still, more randomized controlled clinical trials involving large samples of patients are needed in order to provide more evidence.

The aim of this narrative review was to assess the effect of inulin on IBS-C. Literature searches were conducted in PubMed using the following key-words: Inulin, irritable bowel syndrome, constipation, in order to investigate the clinical use of inulin products in patients suffering of IBS-C. We included all the references found related to the subject published in English until February 2020.

\section{Inulin and stool frequency}

A meta-analysis published in 2014 showed that the intake of inulin has a positive effect on bowel function in individuals with chronic constipation (10). The beneficial effect is reflected by several indicators of the intestinal habits, such as increased number of stools per week, lower stool consistency according to the Bristol scale and lower intestinal transit time. As regarding the stool frequency, the results of this meta-analysis sustain the data obtained by others studies $(11,12)$, that the consumption of inulin contributes to the perception of beneficial effects. A pilot-study conducted in 2013 on IBS-C patients who received yogurt enriched with inulin demonstrated a consistent benefit regarding bowel habits (13).

Similar effects were reported by several other studies regarding increased stool frequency after supplementation with different doses of inulin $(12,14)$. A dose of at least $12 \mathrm{~g}$ of inulin consumed per day leads to significantly higher stool frequency in patients with chronic constipation (12). These data are also sustained by the scientific opinion of the European Food Safety Authority (EFSA), which postulated that there is a cause-effect relationship between the consumption of $12 \mathrm{~g} /$ day of 'native chicory inulin' and the maintenance of normal defecation by increasing stool frequency, without resulting in digestive discomfort (15).

Apparently, the industrial inulin-based products are considered equally beneficial for improving stool habits $(5,12)$.

\section{Inulin and stool consistency}

In clinical trials the consumption of inulin is also connected to the improvement of stool consistency assessed with Bristol Form Scale $(11,12)$. The regular consumption of fermented milk beverages enriched with inulin it is also beneficial showing significant improvement of consistency of stools in patients with IBS-C (13). Usefulness of chicory inulin has been revealed in a placebo-controlled and randomized, double-blind study on subjects with constipation (5). Subjects taking inulin had a softening of stool consistency compared with placebo group. The results of the meta-analysis conducted of Collado Yurrita et al (10) are in agreement with the data above. The inulin ingestion is effective in increasing stool quality in patients presenting with chronic constipation as it reduces the hardness of stools.

\section{Inulin and transit time}

Regarding the effect of inulin on bowel transit time, inulin reduces significantly the transit time through colon as demonstrated by the study of Isakov et al (13). Inulin enriched yogurt had a beneficial effect on transit time in patients with IBS-C when compared with traditional yogurt. However, the number of patients enrolled in the study was small, so additional large double-blinded randomized controlled studies are needed in order to consolidate the role of inulin upon bowel transit time.

\section{Inulin and patients satisfaction}

Improvement of patients satisfaction was seen in subjects with constipation receiving inulin when comparing to placebo (5). This could be explained by the improvement of stool frequency, as some studies demonstrated that abdominal pain and bloating did not improve with inulin intake (10), while several other studies showed that the quality of life depends on reduction of abdominal pain and distention in patients consuming inulin (11).

\section{Inulin and gut microbiota}

Based on the observation that in IBS patients there is an alteration of gut microbiota, studies confirmed that the consumption of inulin has a beneficial effect, as it exerts a stimulatory effect on health-promoting bacteria from genus Bifidobacterium. A double-blind, randomized, cross-over intervention study showed that inulin has a beneficial effect by increasing Bifidobacterium species and lowering those of Bilophila and Anaerostipes, thus confirming the prebiotic role of inulin (16). Inulin stimulates saccharolytic fermentation in cecum and colon as it is fermented by bacteria, producing short chain fatty acids, which are recognized as health promoting metabolites. This statement is sustained also by other studies $(17,18)$. On the contrary, a recent study showed that inulin consumption did not modulate taxa abundance or overall gut microbiota composition significantly (19). This outcome may be due to the small number of subjects, population selected and/or other aspects of the study design, as concluded the authors. The bifidogenic potential of inulin is also demonstrated by a trial 
on healthy volunteer which showed that the consumption of chicory inulin or Jerusalem artichoke stimulated the growth of bifidobacteria and reduced the amount of Clostridium and Bacteroides species (7).

\section{Conclusion}

In conclusion, inulin may have potential beneficial effects on the quality of life and bowel function of IBS-C patients in terms of stool frequency, consistency and transit time. Inulin is well tolerated, lacking side effects if it is consumed in recommended dosage. However, our conclusions are drawn from several studies and may include small samples, non IBS design studies. Large randomized control studies are needed to confirm the effect of inulin in these patients.

\section{Acknowledgements}

Not applicable.

\section{Funding}

No funding was received.

\section{Availability of data and materials}

All data generated or analyzed during this study are included in this published article.

\section{Authors' contributions}

OBB conducted the literature research and wrote the manuscript. ICi, ICh and CA contributed to the literature research and revised the manuscript. VD conducted the literature research, wrote the manuscript and coordinated the study. All authors read and approved the final manuscript.

\section{Ethics approval and consent to participate}

Not applicable.

\section{Patient consent for publication}

Not applicable.

\section{Competing interests}

The authors declare that they have no competing interests.

\section{References}

1. Dahl WJ and Stewart ML: Position of the Academy of Nutrition and Dietetics: Health implications of dietary fiber. J Acad Nutr Diet 115: 1861-1870, 2015.
2. Schmier JK, Miller PE, Levine JA, Perez V, Maki KC, Rains TM, Devareddy L, Sanders LM and Alexander DD: Cost savings of reduced constipation rates attributed to increased dietary fiber intakes: A decision-analytic model. BMC Public Health 14: 374, 2014.

3. David LA, Maurice CF, Carmody RN, Gootenberg DB, Button JE, Wolfe BE, Ling AV, Devlin AS, Varma Y, Fischbach MA, et al: Diet rapidly and reproducibly alters the human gut microbiome. Nature 505: 559-563, 2014.

4. Slavin J: Fiber and prebiotics: Mechanisms and health benefits. Nutrients 5: 1417-1435, 2013.

5. Micka A, Siepelmeyer A, Holz A, Theis S and Schön C: Effect of consumption of chicory inulin on bowel function in healthy subjects with constipation: A randomized, double-blind, placebo-controlled trial. Int J Food Sci Nutr 68: 82-89, 2017.

6. Öhman L and Simrén M: Intestinal microbiota and its role in irritable bowel syndrome (IBS). Curr Gastroenterol Rep 15: 323, 2013.

7. Kleessen B, Schwarz S, Boehm A, Fuhrmann H, Richter A, Henle $T$ and Krueger M: Jerusalem artichoke and chicory inulin in bakery products affect faecal microbiota of healthy volunteers. Br J Nutr 98: 540-549, 2007.

8. Ramnani P, Gaudier E, Bingham M, van Bruggen P, Tuohy KM and Gibson GR: Prebiotic effect of fruit and vegetable shots containing Jerusalem artichoke inulin: A human intervention study. Br J Nutr 104: 233-240, 2010.

9. Morales-Filho JP and Quigley EM: The intestinal microbiota and the role of probiotics in irritable bowel syndrome: A review. Arq Gastroenterol 52: 331-338, 2015.

10. Collado Yurrita L, San Mauro Martín I, Ciudad-Cabañas MJ, Calle-Purón ME and Hernández Cabria M: Effectiveness of inulin intake on indicators of chronic constipation; a meta-analysis of controlled randomized clinical trials. Nutr Hosp 30: 244-252, 2014

11. Gruenwald J, Busch R and Bentley C: Efficacy and tolerability of Laxatan Granulat in patients with chronic constipation. Clin Exp Gastroenterol 2: 95-100, 2009.

12. Hond ED, Geypens B and Ghoos Y: Effect of high performance chicory inulin on constipation. Nutr Res 20: 731-736, 2000.

13. Isakov VA, Pilipenko VI, Shakhovskaya A and Tutelyan V: Efficacy of inulin enriched yogurt on bowel habits in patients with irritable bowel syndrome with constipation: A pilot study. FASEB J 27: lb426, 2013.

14. Gråsten $\mathrm{S}$, Liukkonen $\mathrm{KH}$, Chrevatidis A, El-Nezami H, Poutanen K and Mykkänen H: Effects of wheat pentosan and inulin on the metabolic activity of fecal microbiota and on bowel function in healthy humans. Nutrit Res 23: 1503-1514, 2003.

15. EFSA Panel on Dietetic Products, Nutrition and Allergies: Scientific opinion on the substantiation of a health claim related to 'native chicory inulin' and maintenance of normal defecation by increasing stool frequency pursuant to Article 13.5 of Regulation (EC) No 1924/20061. EFSA J 13: 3951, 2015.

16. Vandeputte D, Falony G, Vieira-Silva S, Wang J, Sailer M, Theis S, Verbeke K and Raes J: Prebiotic inulin-type fructans induce specific changes in the human gut microbiota. Gut 66 : 1968-1974, 2017.

17. Dewulf EM, Cani PD, Claus SP, Fuentes S, Puylaert PG, Neyrinck AM, Bindels LB, de Vos WM, Gibson GR, Thissen JP and Delzenne NM: Insight into the prebiotic concept: Lessons from an exploratory, double blind intervention study with inulin-type fructans in obese women. Gut 62: 1112-1121, 2013.

18. Linetzky Waitzberg D, Alves Pereira CC, Logullo L, Manzoni Jacintho T, Almeida D, Teixeira da Silva ML and Matos de Miranda Torrinhas RS: Microbiota benefits after inulin and partially hydrolized guar gum supplementation: A randomized clinical trial in constipated women. Nutr Hosp 27: 123-129, 2012.

19. Watson AW, Houghton D, Avery PJ, Stewart C, Vaughan EE, Meyer PD, de Bos Kuil MJJ, Weijs PJM and Brandt K: Changes in stool frequency following chicory inulin consumption, and effects on stool consistency, quality of life and composition of gut microbiota. Food Hydrocoll 96: 688-698, 2019. 